\title{
Systematic Approach for Anterior Corpectomy through a Transthoracic Exposure
}

\author{
Varun PUVANESARAJAH*, Ioan A. LINA*, Jason A. LIAUW, Sheng-fu L. LO, Benjamin D. ELDER, \\ Jean-Paul WOLINSKY, Ali BYDON, Daniel M. SCIUBBA, Ziya L. GOKASLAN, Timothy F. WITHAM
}

The Johns Hopkins University, School of Medicine, Department of Neurosurgery, Baltimore, Maryland, USA

*PUVANESARAJAH and LINA contributed equally to this manuscript.

9

To watch the surgical videoclip, please visit http://turkishneurosurgery.org.tr/images/15271-video.mp4

\section{ABSTRACT}

While several approaches have been suggested for performing a transthoracic corpectomy of the thoracolumbar spine, the steep learning curve and high risk of operative morbidity often dissuades its use clinically. As a result, posterior approaches have been more frequently used, though transthoracic approaches often allow for improved access for more complete decompression of the spinal cord. Additionally, the transthoracic approach allows for more complete removal of vertebral body lesions along with better access for reconstruction of the anterior column. Here we describe a distinct nine-step thoracolumbar corpectomy technique via a transthoracic approach. We include a description of one patient with one-year follow-up to further illustrate the surgical approach and relevant surgical anatomy. We have used this technique for maximal bi-pedicular decompression afforded by the lateral approach. We discuss key anatomical considerations, perioperative techniques and potential complications of the operation. The presented nine-step transthoracic corpectomy technique is a safe method for providing adequate neurological decompression and vertebral body tumor removal.

KEYWORDS: Spinal tumor, Corpectomy, Transthoracic, Cage

\section{INTRODUCTION}

A thoracic corpectomy is indicated in various pathologies of the anterior column of the spine including traumatic injury, neoplastic involvement, and osteomyelitis $(8,11,12)$. While the technique of performing a thoracic corpectomy has evolved over the past few decades, the procedure is still associated with significant morbidity due to technical challenges tied to its invasiveness as well as complications incurred with spinal instrumentation.

Based on a patient's unique anatomy and case characteristics, one can choose between several different surgical approaches. Traditionally, an anterior approach was felt to allow the most direct access to the vertebral body, facilitating visualization and anterior column manipulation while avoiding spinal cord injury $(3,17,18)$. This approach provides a significant advantage when treating extensive vertebral body trauma, metastatic disease, or degenerative disease.

However, in the thoracolumbar spine, posterior approaches are often preferred due to surgeon familiarity and procedural morbidity often associated with anterior approaches $(5,6,14)$. Although anterior approaches are less common, they allow for improved access corridors for decompression of the spinal 
cord, and in combination with posterior approaches, allow for circumferential decompression of the spinal cord. Anterior approaches also prove useful in rectifying the compromised load-bearing capacity of the ventral spine caused by osteolytic tumor invasion and pathological fractures, without directly disrupting the posterior elements. Preservation of these posterior elements is important as it helps to maintain stability of the spine.

This manuscript describes a nine-step anterior corpectomy technique, which allows for a full bipedicular ventral decompression of the spinal cord while reducing the risk of surgically related comorbidities. This technique directly addresses the common shortcoming of limited ventral decompression that often occurs with transthoracic corpectomy. From our experience, this technique provides a systematic and safe method for drilling and decompression so as to prevent any iatrogenic cerebrospinal fluid (CSF) leak or spinal cord injury. While this is not a novel technique, the goal of this work is to provide a comprehensive presentation of the approach along with a case study, with a review of surgical anatomy and relevant clinical pearls based on our experience.

\section{CASE REPORT}

A 61-year old female presented with significant upper low back pain (8/10). She was previously diagnosed with breast cancer and imaging revealed a metastatic lesion in the T12 vertebral body (Figure 1A, B). The patient experienced minimal weakness secondary to pain. Various treatment options were explored including radiation therapy. However, it was determined that given instability, radiation would not adequately ameliorate the patient's pain. Thus, a corpectomy with anterior reconstruction was planned.

Adequate tumor debulking was achieved with an anterior corpectomy technique (Figure 2A-C). During the procedure the patient's estimated blood loss was $300 \mathrm{ml}$. Other than pain
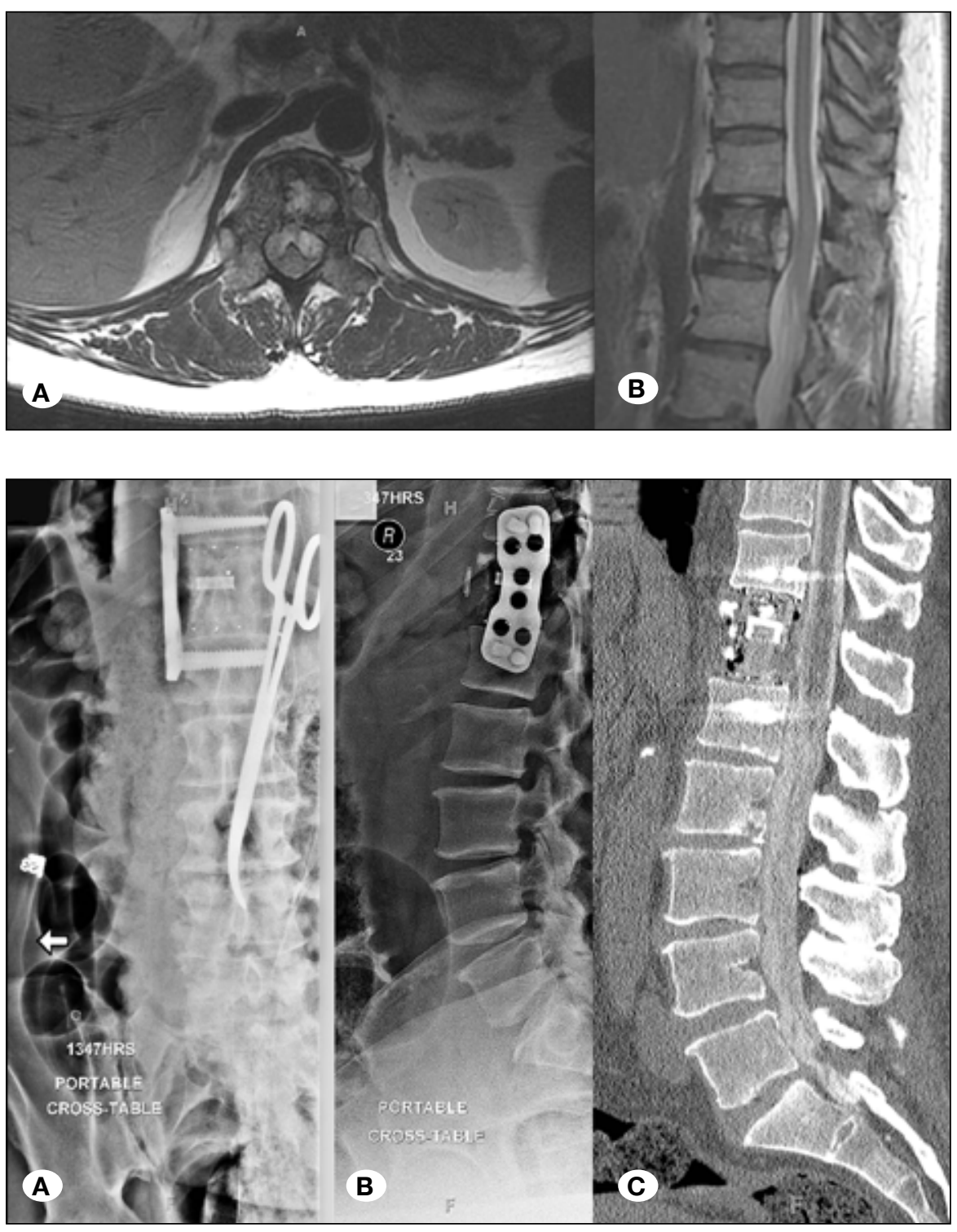

Figure 1: Preoperative images depicting the lesion. A) Axial MRI. B) Sagittal MRI.
Figure 2: Images demonstrating successful corpectomy with cage placement. A) Intraoperative AP X-ray B) Postoperative lateral X-ray C) Postoperative sagittal CT myelogram. 
at the site of chest tube placement, the patient did not have any intraoperative or anesthetic complications. The remainder of the patient's five-day post-operative hospital stay was uncomplicated and her pain was significantly improved at discharge. The patient was treated with stereotactic radiosurgery two weeks after the procedure to ensure local control. Since her procedure, the patient has been pain free with no evidence of local tumor recurrence over the past year.

\section{Operative Technique}

First, we describe the thoracotomy and thoracoabdominal approaches and considerations. This is followed by the full corpectomy description (Video).

\section{Choosing an Approach}

The selection of a right or left sided approach is determined by several factors. If an extravertebral malignancy requires resection, the side with the greatest tumor bulk is chosen for approach while taking into consideration any intrathoracic organ involvement. For instance, if pulmonary function is poor or pulmonary metastases are observed necessitating pulmonary resection, a double lumen endotracheal tube should be used. However, if pulmonary function is normal and no pulmonary metastases are present, a single-lumen endotracheal tube should suffice. In patients who have had previous thoracic operations, the contralateral hemithorax should be selected to mitigate the risk of prolonged postoperative air leaks and increased chest tube time, presumably leading to lower rates of infection.

For T4-T6 thoracotomies, we recommend approaching the spine from the right side, as the descending aorta and aortic arch can complicate the approach from the left side. However, below T6, it is easier to approach from the left side as the liver can limit the approach from the right. Furthermore, the inferior vena cava is present in the right hemithorax and is much more difficult to repair than the aorta should inadvertent vascular injury occur.

\section{Thoracotomy (T3-T11)}

T3 is generally regarded as a transitional level between the thoracic apex and the remainder of the upper thoracic cavity. An anterolateral thoracotomy is recommended for the upper thoracic region from T3 to T10, though the scapula is an important consideration. Above T7, which marks the caudal end of the scapula and corresponds to the bottom of the sixth rib, a transthoracic approach necessitates mobilization of the scapula. To release and elevate the scapula, the patient's arm is fully abducted and internally rotated such that the scapula is elevated, thus preventing obstruction of the surgical corridor. Complete mobilization of the scapula entails isolating and cutting the rhomboids and trapezius muscles off the lower edge of the scapula.

Due to the changing caudal angulation of the ribs from T3$\mathrm{T} 12$, we generally recommend resecting ribs based on the vertebral level of interest, as described in Table I. While these guidelines are useful for planning a thoracotomy, an intraoperative $x$-ray with a radiopaque marker often helps to select the best rib for resection prior to draping. A preoperative $\mathrm{x}$-ray confirms the optimal patient position on the table and facilitates interpretation of subsequent $x$-rays. The intercostal neurovascular bundle and muscles must also be detached before two lateral cuts are made to allow removal of the lateral-most section of the rib (Figure $3 \mathrm{~A}, \mathrm{~B}$ ).

\section{Thoracoabdominal Approach (T12-L2)}

To gain simultaneous access to $\mathrm{T} 12$ and $\mathrm{L} 1$, one needs to gain access to the retroperitoneal space in order to expose adequate vertebral body surface area for plate fixation. To limit the incision size, we use a modified $10^{\text {th }}$ rib approach, where only the posterior portion of the $10^{\text {th }}$ rib is cut enabling detachment of the posterior portion of the diaphragm. By dividing only the posterior most portion of the diaphragm, the chance of accidentally entering the peritoneal cavity is limited, since going through the posterior extension of the diaphragm places the exposure over the retroperitoneal fat. In the case of an iatrogenic peritoneal opening, one can either suture the opening if it is small, or widen the opening to prevent any chance of bowel strangulation if bowel were to herniate through the peritoneal opening.

Upon exposure of the intrathoracic space and diaphragm, the ipsilateral lung is deflated and retracted allowing access to the retroperitoneal cavity following the detachment of the posterior portion of the diaphragm. The requisite surgical manipulation of the diaphragm has been reviewed in detail previously (1). It should be highlighted that the diaphragm is innervated centrifugally; therefore, any cut through the diaphragm leads to paralysis of the remaining muscle cuff extending radially from the cut. A two-inch diaphragmatic cuff is retained to facilitate reapproximation at the time of closure. The diaphragmatic incision should extend directly to the midpoint of its insertion on the vertebral body of T12 (Figure 3C, D).

\section{Corpectomy (Table II, Figure 4)}

After visualization and confirmation of the correct level with a localizing needle, the parietal pleura is incised and reflected ventrally. For procedures where partial corpectomies are required to remove an intervertebral disc, our institution has advocated utilization of a technique of polymethylmethacrylate marking of the vertebral body for elective cases in the thoracic spine. This technique helps to facilitate radiographic localization of the correct level without the need to count

Table I: Guidelines for Rib Removal by Targeted Vertebral Level

\begin{tabular}{ll}
\hline $\begin{array}{l}\text { Vertebral body of } \\
\text { primary involvement }\end{array}$ & Removed rib \\
\hline T3, T4 & $4^{\text {th }}$ \\
\hline T5, T6 & $5^{\text {th }}$ \\
\hline T7, T8 & $\begin{array}{l}\text { One level above (for T7, } \\
\text { remove } 6^{\text {th }} \text { rib, etc.) }\end{array}$ \\
\hline T9-T12 & $\begin{array}{l}\text { Two levels above (for T9, } \\
\text { remove } 7^{\text {th }} \text { rib, etc.) }\end{array}$ \\
\hline
\end{tabular}




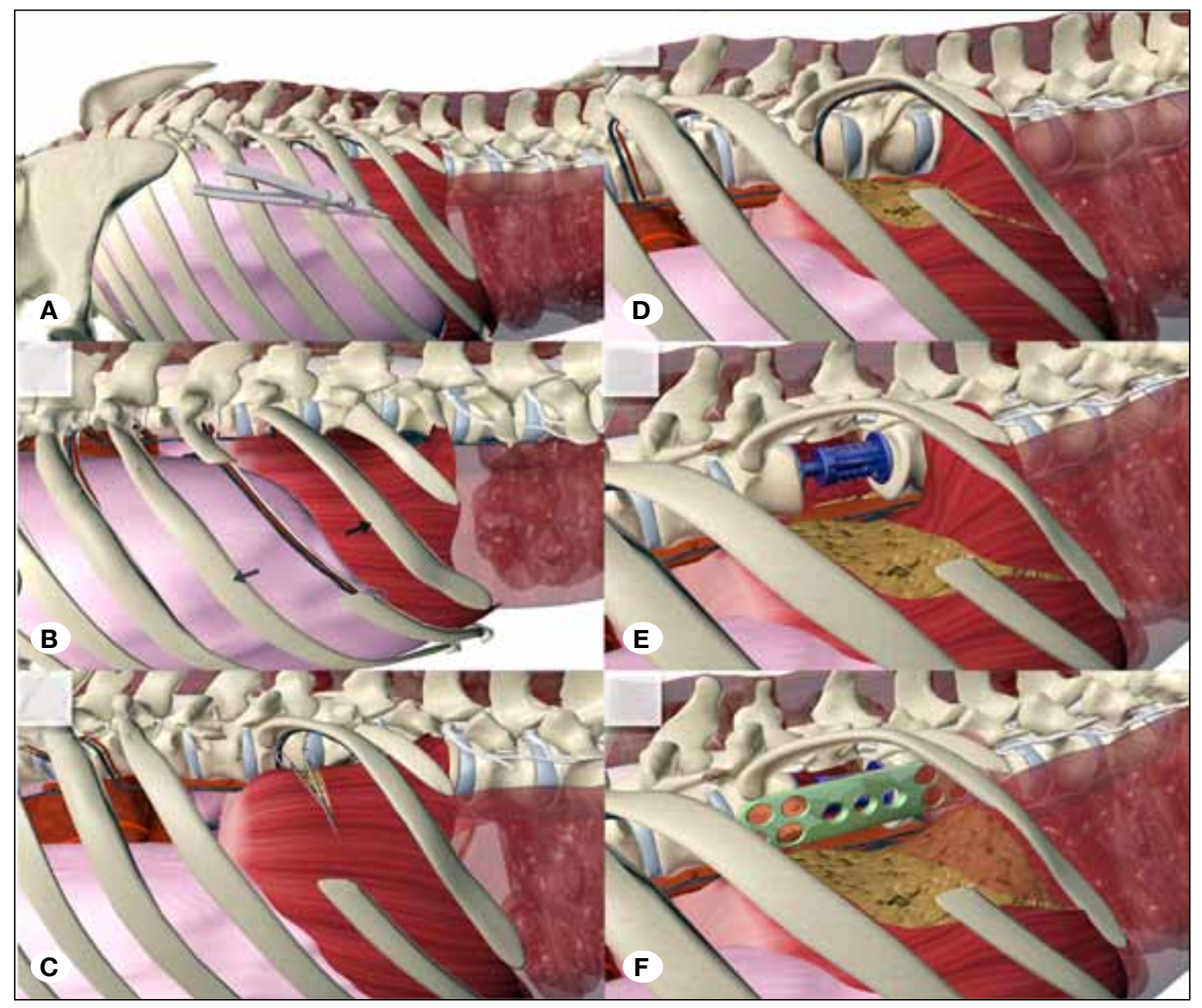

Figure 3: 3D computer-assisted designs demonstrating key steps of the thoracoabdominal approach. A) Cutting of the rib; B) Retraction of the ribs; C) Cutting the diaphragm; D) Retraction of retroperitoneal fat; E) Placement of an expandable cage; F) Placement of anterior plate and screws.

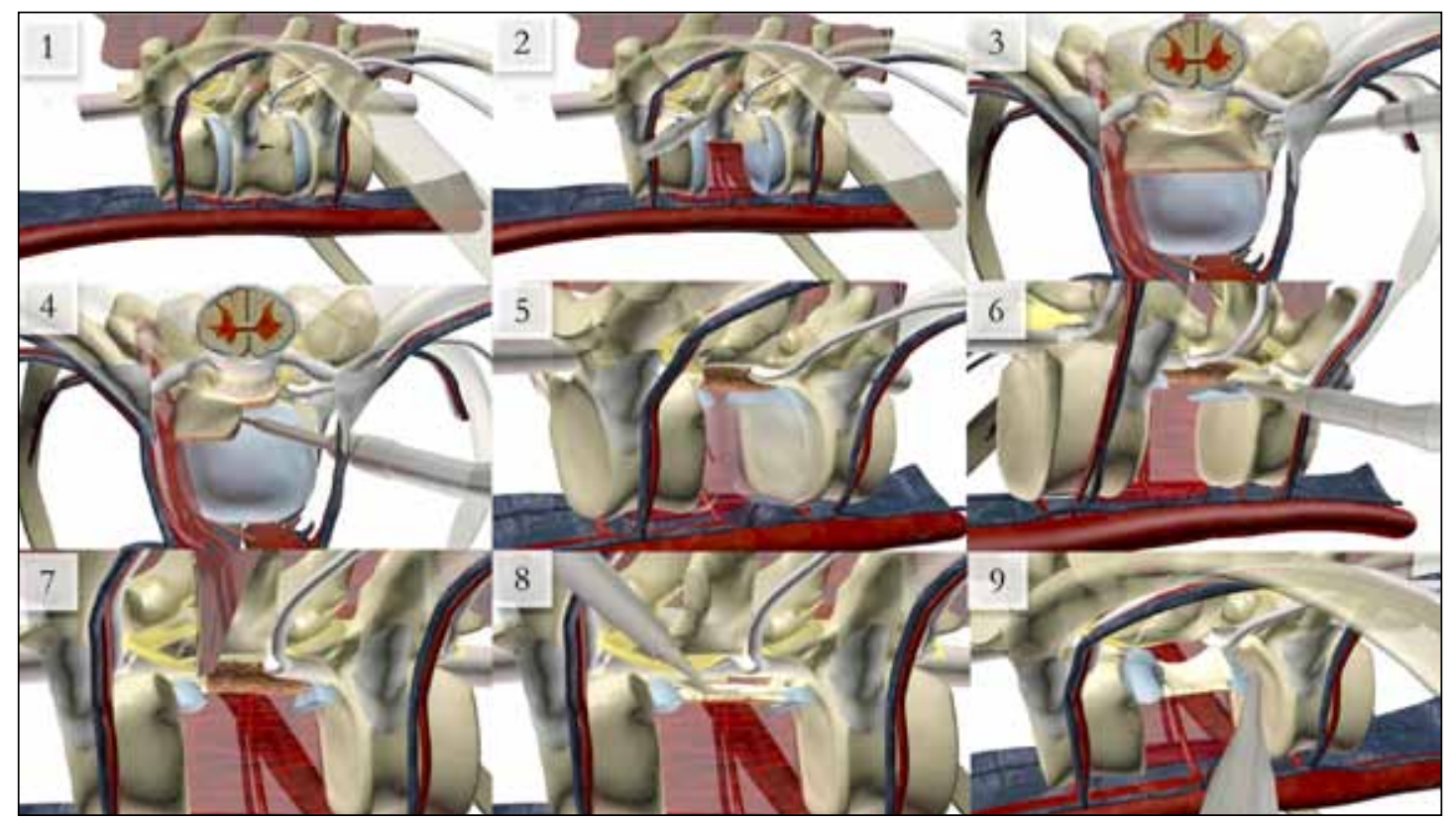

Figure 4: 3D computer-assisted designs describing each step of the nine-step corpectomy technique as detailed in Table II. 
Table II: Summary of Nine-Step Corpectomy Technique

\begin{tabular}{cl}
\hline Step & Description \\
\hline 1 & Make a rectangular cut into anterior vertebral body. \\
\hline 2 & $\begin{array}{l}\text { Electrocauterize around the rib head to sever the remaining muscle attachments and remove rib head, thus exposing } \\
\text { the ipsilateral pedicle. }\end{array}$ \\
\hline 3 & Drill out ipsilateral pedicle, being careful to leave the shell of bone on the medial aspect of the pedicle. \\
\hline 4 & $\begin{array}{l}\text { Drill the contralateral pedicle, likewise ensuring to leave a cortical shell of bone on the medial aspect of the } \\
\text { contralateral pedicle. }\end{array}$ \\
\hline 6 & $\begin{array}{l}\text { Remove bulk of the inferior and superior intervertebral disks to expose adjacent endplates. } \\
\text { superior endplate and PLL. }\end{array}$ \\
\hline 8 & $\begin{array}{l}\text { Bone should be removed from the vertebral body in a piecemeal fashion with a Kerrison punch, working in a superior } \\
\text { to inferior direction. }\end{array}$ \\
\hline 9 & Remove the ipsilateral and contralateral pedicle cortical remnants and the PLL. \\
\hline
\end{tabular}

levels from the sacrum or cervical spine to the level of interest (11). Ligation and transection of segmental vessels at the corpectomy level and those levels above and below the surgical site, that will ultimately hold fusion instrumentation, is then performed. At this point, the anterolateral face of the target vertebral level should be completely exposed.

The technique for a complete corpectomy and bilateral pedicular decompression consists of nine steps (Figure 4). First, a rectangular cut is made into the anterior vertebral body with either an ultrasonic bone scalpel or a high-speed drill (Step 1). Next, an electrocautery tool is used to sever any remaining muscle attachments from the rib head, which is subsequently removed with either a drill or rongeur (Step 2). The subcortical bone of the ipsilateral pedicle is then drilled out with either a matchstick or diamond burr while being careful to leave a thin shell of bone on the medial aspect of the pedicle (Step 3). A matchstick burr is often used to core out a majority of the cancellous bone and then a larger diamond burr is used when approaching the cortical shell. The contralateral pedicle is then similarly drilled (Step 4). However, it can be more technically challenging given that drilling posteriorly along the contralateral pedicle requires angulation of the drill. The thin layer of bone is retained on the medial aspect of the pedicle to protect the cord from injury during drilling. Once the pedicles are reduced, the bulk of the inferior and superior intervertebral disks are resected to expose adjacent endplates, leaving behind small nubs at the posterior longitudinal ligament (PLL) attachment to the endplates (Step 5).

The superior endplate is then drilled with a diamond burr at the precise location where the PLL attaches to the superior endplate (Step 6), allowing for future detachment of the PLL. At this point, the remaining cortical shell of the vertebral body and the PLL can be removed in a piecemeal fashion with a Kerrison punch, beginning at the entry site adjacent to the superior endplate that has already been exposed with the diamond burr (Step 7). This start site is important as it allows cranial positioning above the shoulder of the nerve root. All Kerrison bites are directed from the superior to inferior direction so as to limit the probability of contacting the nerve root.

A nervehook is subsequently used to slowly isolate the PLL from the dura (Step 8). After this step is completed, the remaining cortical bone fragments of the ipsilateral and contralateral pedicles, the PLL, and the remaining intervertebral discs, are removed all at once, completing the corpectomy (Step 9)

In the case of an oncological resection, the PLL may be infiltrated by tumor, and this maneuver will increase the chance of a gross total resection. Occasionally the Cavitron Ultrasonic Aspirator (ValleyLab, Inc, Boulder, CO) is useful when the tumor consistency is too soft for the high-speed drill, yet too solid for regular suction devices. Additionally, patients who have had prior radiation treatments may also present with PLL adhesions to the dura. In these cases, given the higher risk of CSF leak, it is important to consider the use of muscular flaps as an additional barrier to prevent CSF drainage into the lungs. At the completion of resection, the endplates of the vertebral bodies above and below should be fully visualized and free of both disc and gross tumor.

The procedure is finalized with anterior reconstruction of the defect. This can be accomplished with an expandable cage, a mesh cage, a bone graft, or polymethylmethacrylate cement. The use of an expandable cage with additional anterior plate placement for translational stability is demonstrated in figures $3 E$ and $3 F$. The rib head can serve as a good landmark for anterior plate placement, particularly at cephalad thoracic levels (13).

Once the corpectomy is complete, the surgeon should check for potential air leaks of the pleural cavity to prevent pneumothorax. Additionally, a chest tube should be placed at the apex of the lung to allow trapped air and blood to escape. 


\section{DISCUSSION}

The presented anterior approach allows for direct visualization of the anterior column, which is necessary for successful implementation of the corpectomy technique described above. This technique is highly effective in ensuring full removal of a vertebral body and both pedicles, which is the most complete method for neurological decompression short of a full $360^{\circ}$ approach. Although this technique has been described previously, this work provides a detailed description and analysis of the approach, to serve as a surgical guide for mastery of this complex procedure. These approaches have been previously reviewed by Fourney and Gokaslan (7) but have been included here as mastery of this approach is necessary for successful transthoracic corpectomy.

Although this technique is very useful, it may be relatively contraindicated in certain patient populations. For example, anterior thoracic approaches should not be used in patients with lungs that are too frail to accommodate positive pressure ventilation. Other contraindications include previous breach of the thoracic cavity and hemodynamic instability (4).

Additionally, there are specific perioperative complications observed with anterior approaches that are not seen with posterior approaches. Not surprisingly, an increased risk of pulmonary complications has been observed. Such complications include atelectasis, pulmonary embolus, pneumothorax, and pleural effusion $(5,6)$. Intercostal neuralgia and significant pain has also been reported (14). The overall complication rate for the anterior approach has been estimated to be around $11-11.5 \%(5,6)$. Another issue is that breach of the dura and resultant CSF leak is a reported occurrence that can complicate these procedures. A CSF-pleural fistula can occur, which may ultimately require re-exploration of the surgical site and prove difficult to repair (15). Some of the clinical pearls illustrated in this manuscript may help to avoid this complication, thus reducing a significant source of morbidity in transthoracic corpectomy patients.

Some of these complications also could potentially be avoided by employing a posterior approach, though the posterior approach has its own inherent limitations and complications. Posterior approaches significantly limit a surgeon's working space, are technically demanding, and can result in cord injury (10). They also limit the amount of anterior decompression that can be performed, as minimal manipulation of the thoracic spinal cord can safely be performed. Furthermore, posterolateral approaches, such as the lateral extracavitary approach, have been associated with excessive blood loss and postoperative pain due to extensive muscle manipulation $(2,9,16)$. In general, we recommend choosing a surgical approach that best addresses the location of the surgical pathology. For instance, in the thoracic spine, the transthoracic approach provides the safest corridor for pathology ventral/ medial to the thecal sac, as no manipulation of the spinal cord is needed. A costotransversectomy can also be used, though in a costotransversectomy approach it can be challenging to adequately decompress pathology immediately ventral to the spinal cord. A transpedicular approach does not typically allow for any access directly ventral to the spinal cord. Finally, a midline posterior approach is only useful for addressing pathology dorsal to the spinal cord in the thoracic spine.

\section{CONCLUSION}

We have described an effective technique for anterior thoracic corpectomies. A key benefit of the presented approach and corpectomy technique is that it allows for full spinal cord protection while fully removing the vertebral body, thus allowing for adequate neurological decompression and tumor or bone fragment removal in a safe manner.

\section{- ACKNOWLEDGMENTS}

Benjamin D. Elder: This article reflects the views of the author and should not be construed to represent the FDA's views or policies.

C. Rory Goodwin: Supported by the UNCF-Merck Postdoctoral Fellowship and an Award from the Burroughs Wellcome Fund

Ali Bydon: Recipient of a research grant from DePuy Spine and serves on the clinical advisory board of Medlmmune, LLC

Ziya L. Gokaslan: Disclosures of stock ownership in US Spine and Spinal Kinetics, consulting, speaking and teaching for the AO Foundation and research support from DePuy, NREF, AO Spine, and AO North America

Timothy F. Witham: Recipient of research funding from Eli Lily and Company; research support from the Gordon and Marilyn Macklin Foundation

Daniel M. Sciubba: Consultant for DePuy, Medtronic, Nuvasive, and Globus

\section{- REFERENCES}

1. Baaj AA, Papadimitriou K, Amin AG, Kretzer RM, Wolinsky JP, Gokaslan ZL: Surgical anatomy of the diaphragm in the anterolateral approach to the spine: a cadaveric study. J Spinal Disord Tech 27: 220-223, 2014

2. Benzel EC: The lateral extracavitary approach to the spine using the three-quarter prone position. J Neurosurg 71: 837841,1989

3. Chen LH, Chen WJ, Niu CC, Shih $\mathrm{CH}$ : Anterior reconstructive spinal surgery with Zielke instrumentation for metastatic malignancies of the spine. Arch Orthop Trauma Surg 120: $27-$ 31,2000

4. Donahue JM, Smith MA, Battafarano RJ: Complications of thoracoscopy. In: Mulholland MW, (ed). Complications in Surgery. Philadelphia: Wolters Kluwer/Lippincott Williams \& Wilkins, 2011:320-327

5. Faciszewski T, Winter RB, Lonstein JE, Denis F, Johnson L: The surgical and medical perioperative complications of anterior spinal fusion surgery in the thoracic and lumbar spine in adults. A review of 1223 procedures. Spine (Phila Pa 1976) 20: 1592-1599, 1995

6. Fessler RG, Sturgill M: Review: Complications of surgery for thoracic disc disease. Surg Neurol 49: 609-618, 1998 
7. Fourney DR, Gokaslan ZL: Anterior approaches for thoracolumbar metastatic spine tumors. Neurosurg Clin N Am 15: 443-451, 2004

8. Gokaslan ZL, York JE, Walsh GL, McCutcheon IE, Lang FF, Putnam JB Jr, Wildrick DM, Swisher SG, Abi-Said D, Sawaya $\mathrm{R}$ : Transthoracic vertebrectomy for metastatic spinal tumors. J Neurosurg 89: 599-609, 1998

9. Graham AW 3rd, Mac Millan M, Fessler RG: Lateral extracavitary approach to the thoracic and thoracolumbar spine. Orthopedics 20: 605-610, 1997

10. Hofstetter CP, Chou D, Newman CB, Aryan HE, Girardi FP, Hartl $\mathrm{R}$ : Posterior approach for thoracolumbar corpectomies with expandable cage placement and circumferential arthrodesis: A multicenter case series of 67 patients. J Neurosurg Spine 14: 388-397, 2011

11. Hsu W, Sciubba DM, Sasson AD, Khavkin Y, Wolinsky JP, Gailloud P, Gokaslan ZL, Murphy K: Intraoperative localization of thoracic spine level with preoperative percutaneous placement of intravertebral polymethylmethacrylate. J Spinal Disord Tech 21: 72-75, 2008

12. Kim DH, O'Toole JE, Ogden AT, Eichholz KM, Song J, Christie SD, Fessler RG: Minimally invasive posterolateral thoracic corpectomy: Cadaveric feasibility study and report of four clinical cases. Neurosurgery 64:746-752; discussion 752-753, 2009
13. Papadimitriou K, Amin AG, Kretzer RM, Chaput C, Tortolani PJ, Wolinsky JP, Gokaslan ZL, Baaj AA: The rib head as a landmark in the anterolateral approach to the thoracic spine: A computed tomography-based morphometric study. J Neurosurg Spine 18: 484-489, 2013

14. Payer M, Sottas C: Mini-open anterior approach for corpectomy in the thoracolumbar spine. Surg Neurol 69:2531; discussion 31-32, 2008

15. Raffa SJ, Benglis DM, Levi AD: Treatment of a persistent iatrogenic cerebrospinal fluid-pleural fistula with a cadaveric dural-pleural graft. Spine J 9: e25-29, 2009

16. Resnick DK, Benzel EC: Lateral extracavitary approach for thoracic and thoracolumbar spine trauma: Operative complications. Neurosurgery 43:796-802; discussion 802803, 1998

17. Rosenthal D, Marquardt G, Lorenz R, Nichtweiss M: Anterior decompression and stabilization using a microsurgical endoscopic technique for metastatic tumors of the thoracic spine. J Neurosurg 84: 565-572, 1996

18. Sundaresan N, Shah J, Foley KM, Rosen G: An anterior surgical approach to the upper thoracic vertebrae. J Neurosurg 61: 686-690, 1984 